\title{
Calculation of the Green's function from high- and low-density series expansions for disordered transport
}

\author{
Daniel F. Calef \\ Department of Chemistry, Massachusetts Institute of Technology, \\ Cambridge, Massachusetts 02139 \\ Richard Friesner \\ Department of Chemistry, University of Texas, Austin, Texas 78712 \\ Gregory Korzeniewski \\ Department of Chemistry, Massachusetts Institute of Technology, \\ Cambridge, Massachusetts 02139 \\ Brian Laird \\ Department of Chemistry, University of Texas, Austin, Texas 78712 \\ Robert Silbey \\ Department of Chemistry, Massachusetts Institute of Technology, \\ Cambridge, Massachusetts 02139
}

(Received 5 March 1984)

\begin{abstract}
We investigate density expansions for the configurationally averaged Green's function for a random walk on a (site) disordered lattice. Two-point Padé summation techniques are used in conjunction with scaling arguments to examine behavior near the percolation density. Recent proposals for the structure of the percolation cluster are discussed in light of the results.
\end{abstract}

The problem of transport of a localized particle in a random network has been the subject of many investigations, ${ }^{1-10}$ which have been applicable either to very long or very short times. In this paper, we present a new calculation which bridges the gap between short and long times, by using both low- and high-density expansions in a novel two-point Padé approximant technique. ${ }^{11}$ As an example, we compute the probability of remaining on the initial site, $P_{0}(t)$, as a function of time for a two-dimensional triangular lattice. Using scaling arguments based on the recent results of Coniglio ${ }^{8}$ for the structure of the percolating cluster, we examine both the critical and noncritical parts of $P_{0}(t)$ near the percolation edge, and finally we address the question of anomalous diffusion on this cluster. ${ }^{10}$

The model we consider is a triangular lattice with randomly populated sites, on which the transport is governed by a master equation with transfer matrix $\underline{W}$, whose elements $W_{u j}=w \xi_{i} \xi_{j}$. Here, $w$ is the nonrandom nearest-neighbor jump rate and $\xi_{i}$ is a random variable for occupation of site $i$ : $\xi_{i}=0$ if $i$ is unoccupied, $\xi_{i}=1$ if it is occupied, and $\left\langle\xi_{i}\right\rangle=\rho$ (the concentration of occupied sites), where the bracket represents an average over all configurations consistent with $\rho$. The probability of occupation of a site at time $t$ is governed by the averaged Green's function, $\underline{G}(t) \equiv\langle\exp \underline{W}\rangle$, or by its Laplace transform, $\underline{G}(u)$ $=\left\langle[u \underline{1}-\underline{W}]^{-1}\right\rangle$. The probability that the particle is at the initial site is given by $G_{00}(t)$, which is, of course, a function of $\rho$. We write the low-density expansion of $\underline{G}(u)$ as

$$
\begin{aligned}
& \underline{G}(u)=\sum_{n=0}^{\infty} \underline{c}^{(n)}(u) \rho^{n}, \\
& \underline{c}^{(n)}(u)=\sum_{\substack{\text { connected } \\
n \rightarrow \text { clusters }}}\left\{\left[u \underline{1}-\underline{W}_{\mathrm{cl}}\right]^{-1}-\sum_{\substack{\text { connected } \\
\text { decompositions }}}\left[u \underline{1}-\underline{W}_{\mathrm{cd}}\right]^{-1}\right\} .
\end{aligned}
$$

The first term on the right-hand side of Eq. (2) corresponds to solving the master equation on a given cluster of $n$ sites; the second term removes from this all processes involving fewer than $n$ sites, leaving only processes contributing to $\rho^{n}$. All possible locations of the $n$ cluster are summed over in Eq. (2). To compute $G_{00}(u)$, we only consider connected clusters containing the origin and sum over all locations by taking the trace for each unique cluster. We enumerate the clusters and perform the matrix inversions on a computer.

In the same spirit, we write the high-density expansion as

$$
\underline{G}(u)=\sum_{n=0}^{\infty} \underline{d}^{(n)}(1-\rho)^{n} \text {, }
$$

where the $\underline{d}^{(n)}$ are found by solving the same master equation on a lattice with $n$ impurities correcting for lower-order processses in the same manner as before.

For the system we are considering, we may write $G_{00}(u)=S(\rho) / u+H(\rho, u)$, where $S(\rho)=\lim _{t \rightarrow \infty} G_{00}(t)$, i.e., $S(\rho)$ is the probability of being on the original site at $t=\infty$. This is not zero (even in an infinite system) since there are finite clusters for all $\rho<1$. We expect that in a finite cluster with $N_{\mathrm{cl}}$ sites, the probability of being on the initial site at $t=\infty$ is $1 / N_{\mathrm{cl}}$, so that $S(p)=\left\langle 1 / N_{\mathrm{cl}}\right\rangle$. We also expect that $S(\rho)$ will be a continuous function of $\beta$. The function $H(\rho, u)$ contains the dynamics, and, in contrast, we expect it to have nonanalytic behavior at $\rho=\rho_{c}$.

In order to calculate $S(\rho)$, we resort to a two-point Padé procedure (TPPA) ${ }^{11}$ using 12 low- and 12 high-density coefficients. The result is shown in Fig. 1. This result is fully converged, and is the most complete calculation of this quantity to this time.

In order to use the TPPA for $H(\rho, u)$ we must justify the fact that such a procedure will inevitably yield the same nonanalytic behavior for $H(\rho, u)$ above and below $\rho_{c}$. We resort to a scaling argument: For very long times (or small 


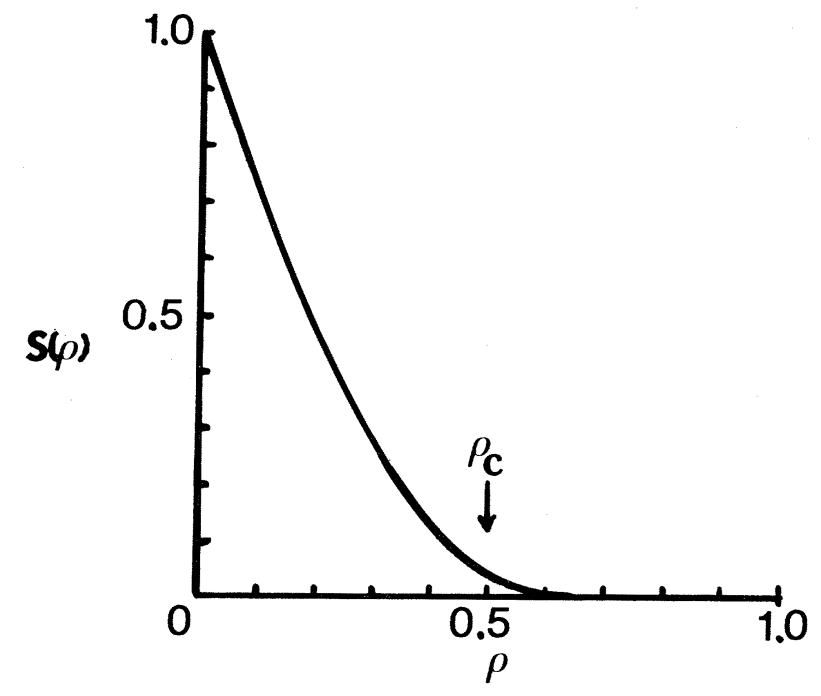

FIG. 1. $S(\rho)$, or $G_{00}(t)$ at $t=\infty$, calculated with the use of two-point Padé approximants.

$u$ ) we expect (in two dimensions) that the first few terms of $H(\rho, u)$ are given by

$$
\begin{aligned}
& H^{-}(\rho, u) \sim A(\rho)+B(\rho) u, \quad \rho<\rho_{c}, \\
& H^{+}(\rho, u) \sim f(\rho) \ln (u)+g(\rho)+h(\rho) u, \quad \rho>\rho_{c} .
\end{aligned}
$$

That is, above $\rho_{c}$ we expect ordinary diffusive motion at long times. We expect that $f(\rho) \sim\left(\rho-\rho_{c}\right)^{-\mu}$ since it should be approximately proportional to the inverse of the diffusion constant which goes to zero at $\rho_{c}$. However, it is expected that for intermediate time, such that the particle has not transported a length equal to the scaling of correlation length $\xi$ (i.e., $u>\xi^{-\theta}$, where $\theta=2$ for diffusive motion), $H(\rho, u) \sim u^{-\phi}$ and independent of $\xi$, both below and above $\rho_{c}$. In order to build this into a scaling form valid above and below $\rho_{c}$ we postulate (note that $\left.\xi \sim\left|\rho-\rho_{c}\right|^{-\nu}\right)$

$$
\begin{aligned}
& H^{-}(u) \sim \xi^{a}\left(1+u \xi^{+\theta}\right)^{-b}, \quad \rho<\rho_{c}, \\
& H^{+}(u) \sim \xi^{\mu / \nu} \ln \left[1+\left(u \xi^{+\theta}\right)^{-c}\right], \quad \rho>\rho_{c} .
\end{aligned}
$$

For $u \xi^{\theta}<<1$, these forms must agree with Eqs. (4) and (5); thus $A \sim \xi^{a} B \sim \xi^{a+\theta}$. For $u \xi^{\theta}>1$, these must both $\sim u^{-\phi}$, independent of $\xi$; thus $a=\theta b, u / \nu=\theta c$, and $b=c=\phi$. We therefore conclude that $A(\rho)$ and $f(\rho)$ in Eqs. (4) and (5) diverge with the same power of $\left|\rho-\rho_{c}\right|$ and can be combined in a TPPA.

We have calculated nine low-density of three high-density $u$-dependent coefficients for the two-dimensional triangular lattice. For intermediate and small $u$, we find that the coefficients take the form

$$
\begin{aligned}
& c_{00}^{n}(u) \sim \alpha_{n} u^{-1}+a_{n}+b_{n} u, \\
& d_{00}^{n}(u) \sim \alpha_{n}^{\prime} u^{-1}+f_{n} \ln (u)+g_{n} .
\end{aligned}
$$

In order to extract the critical behavior, we apply the TPPA method to $\partial \ln H(\rho, u) / \partial \rho$. We find that various sequences converge to $\rho_{c}=0.5$ and $\mu=1.38 \pm 0.02$. From an analysis of $\partial / \partial \rho \ln [\partial / \partial u H(\rho, u)]$, we find $B(\rho) \sim\left|\rho-\rho_{c}\right|^{-\epsilon}$, with $\epsilon=3.8 \pm 0.2$; however, because of numerical difficulties this value should be viewed as provisional.

These results can be understood in terms of the following picture. For $\rho<\rho_{c}$ all the particles are localized in finite clusters, thus

$$
H^{-}(u)=\sum_{n} \frac{W_{n}}{u+1 / \tau_{n}},
$$

where the sum is over all modes of all clusters. Here $\tau_{k}$ is the relaxation time of the mode and $W_{k}$ the weight of this mode in the sum. Near $\rho_{c}$, the longest relaxation times occur in the largest clusters and, since the process is diffusive, $\tau_{\max } \sim \xi^{2}$. To compute $W_{\max }$, note that it must be the product of the probability of being in the largest cluster $\left[\sim\left(\rho_{c}-\rho\right)^{\beta}\right]$ and the relative weight of $\tau_{\max }$ within the largest cluster. The latter is the ratio of the number of slow modes [which should be proportional to the number of "cutting sites," which Coniglio ${ }^{8}$ shows to be proportional to $\left(\rho_{c}-\rho\right)^{-1}$ ] to the total number of modes [which should be proportional to the number of sites which is proportional to $\left(\rho_{c}-\rho\right)^{-\gamma}$. Combining, we find $W_{\max } \sim\left(\rho_{c}-\rho\right)^{\beta-1+\gamma}$. Substituting this into Eq. (10) and expanding for small $u$, we find

$$
H^{-} \sim A\left(\rho_{c}-\rho\right)^{\beta-1+\gamma-2 \nu}+B u\left(\rho_{c}-\rho\right)^{\beta-1+\gamma-4 \nu}+\cdots .
$$

With the use of previously reported values for the various exponents, ${ }^{9} \quad \beta-1+\gamma-2 \nu \sim-1.3$ and $\beta-1+\gamma-4 \nu$ $\sim-4.0$, in good agreement with the values quoted above $(-1.38$ and -3.8$)$.

We can use these results in another way which allows us to examine the finite time behavior. Let us assume that the $c_{00}^{(n)}$ can be approximated by a single term of the form used in Eq. (10), so that

$$
H(\rho, u)=\sum_{n} \rho^{n} \frac{W_{n}}{u+1 / \tau_{n}},
$$

where the sum is over the number of sites in the cluster. Here we have assumed that for every $n$ there is only one effective (very long) relaxation time with weight $W_{n}$. By comparing the small- $u$ behavior of Eq. (12) with our TPPA results, we can find the form of $W_{n}$ and $\tau_{n}$ for large $n$. By comparing the term independent of $u$ and linear in $u$ in Eq. (12) with Eq. (4), we find

$$
\begin{aligned}
& \sum_{n} W_{n} \tau_{n} \rho^{n}=\left(\rho_{c}-\rho\right)^{-1.38} \\
& \sum_{n} W_{n} \tau_{n}^{2} \rho^{n}=\left(\rho_{c}-\rho\right)^{-3.8}
\end{aligned}
$$

By expanding the right-hand side of Eqs. (13a) and (13b), we find for large $n$

$$
\begin{aligned}
& W_{n} \tau_{n} \sim \rho_{c}^{-1.38-n_{n}+0.38}, \\
& W_{n} \tau_{n}^{2} \sim \rho_{c}^{-3.8-n} n^{+2.8} .
\end{aligned}
$$

Solving for $W_{n}$ and $\tau_{n}$, we find at $\rho_{c}$

$$
\lim _{\rho \rightarrow \rho_{c}} G_{00}(t)=\lim _{\rho \rightarrow \rho_{c}} \sum_{n} \rho^{n} W_{n} e^{-t / \tau} \sim t^{-0.4},
$$

i.e., $\phi=0.6$ in the anomalous diffusion regime mentioned above. Note that this is consistent with the values of $a, b$, and $\theta$ found by comparing Eqs. (6) and (7) with our numerical results. This result is for the average over all cluster sizes at $\rho_{c}$. Had we restricted our attention to only the in- 
finite cluster, used the suggestive form

$$
G_{00}^{\mathrm{inf}}=\lim _{n \rightarrow \infty} W_{n} f\left(t / \tau_{n}\right),
$$

and demanded that this remained finite as $n \rightarrow \infty$, we find $G_{00}^{\text {inf }} \sim t^{-0.8}$. This estimate shows that averages over the infinite cluster and those over all the clusters (i.e., the observed behavior) differ as was recently stressed by Gefen, Aharony, and Alexander. ${ }^{10}$

The last question is whether the exponent of $\left(\rho-\rho_{c}\right)$ in $f(\rho)$ [see Eq. (5)] can be identified with the conductivity exponent. In the last year, this exponent (in two dimensions) has been calculated to be $1.26,{ }^{12} 1.28,{ }^{13}$ and conjectured to be $91 / 72=1.264 .{ }^{14}$ In the present calculation of $P_{0}(t)$, we find an exponent of 1.38 . Even though we question whether a discrepancy of less than $10 \%$ is significant (especially in light of the most recent scaling work ${ }^{15}$ which suggests the exponent is $\frac{4}{3}$ ), there are a number of possible explanations for such a discrepancy. The first is that the two-point Padé is inaccurate, i.e., we have too few points or that the series contains "confluent singularities" which seem to cause difficulties with Padé approximants. ${ }^{16}$ The second is that the scaling argument suggesting that $A(\rho)$ and $f(\rho)$ [see Eqs. (4) and (5)] have the same diverging power of $\left|\rho-\rho_{c}\right|$ is not valid. This would imply that we are forcing an erroneous fit which causes the error. Neither of these arguments can be completely ruled out; however, the numerical stability and convergence of our fit suggests that neither is correct.

Another possibility is that for the percolating cluster, the divergence of the inverse of $D$ (or the conductivity) and the divergence in $P_{0}$ are not the same. Since both of these are quantities averaged over all configurations, or over a rather complex distribution function, there is no guarantee that the inverse of the average of diffusion constant is the same as the average of the inverse of the diffusion constant. Thus the functional dependence $P_{0}$ on $\left(\rho-\rho_{c}\right)$ as $\rho \rightarrow \rho_{c}$ need not be exactly the same as that of $\langle D\rangle^{-1}$. Finally, it has recently been suggested that $P_{0}$ be interpreted in terms of the "spectral dimension" of the percolating cluster. ${ }^{14}$

In conclusion, we have presented a new numerical procedure, based on two-point Padé approximants, which yields results for both the critical and noncritical dynamics of the transport of a localized particle on a random network.
${ }^{1}$ H. Scher and M. Lax, Phys. Rev. B 7, 449 (1973); S. W. Haan and R. Zwanzig, J. Chem. Phys. 68, 1879 (1978); J. Klafter and R. Silbey, Phys. Rev. Lett. 44,55 (1980).

${ }^{2}$ Proceedings of a Symposium on Random Walks and Their Application to the Physical and Biological Sciences, edited by G. H. Weiss and R. J. Rubin [J. Stat. Phys. 30, 249 (1983)].

${ }^{3}$ T. Odagaki and M. Lax, Phys. Rev. B 24, 5284 (1981); I. Webman, Phys. Rev. Lett. 47, 1496 (1981); G. Korzeniewski, R. Friesner, and R. Silbey, J. Stat. Phys. $\underline{31}, 451$ (1983); R. F. Loring, H. C. Anderson, and M. D. Fayer, Phys. Rev. Lett. 무, 1324 (1983).

4J. P. Straley, J. Phys. C 13 , 2991 (1980); C. K. Harris and R. B. Stinchcombe, Phys. Rev. Lett. 므, 1399 (1983).

${ }^{5}$ R. B. Stinchcombe and B. P. Watson, J. Phys. C 9, 3221 (1976).

${ }^{6}$ C. D. Mitescu, H. Ottavi, and J. Roussenq, in Electrical Transport and Optical Properties of Inhomogeneous Media-1977, edited by J. C. Garland and D. B. Tanner, AIP Conf. Proc. No. 40 (AIP,
New York, 1978).

${ }^{7}$ R. Fisch and A. B. Harris, Phys. Rev. B 18, 416 (1978).

${ }^{8}$ A. Coniglio, J. Phys. A 15,3829 (1982).

${ }^{9}$ D. Stauffer, Phys. Rep. 54, 3 (1979).

${ }^{10}$ Y. Gefen, A. Aharony, and S. Alexander, Phys. Rev. Lett. $\underline{50}, 77$ (1983).

${ }^{11}$ See, for example, C. M. Bender and S. A. Orszag, Advanced Mathematics for Scientists and Engineers (McGraw-Hill, New York, 1978), Chap. 8.

${ }^{12}$ M. Sahimi, B. Hughes, L. Scriven, and H. T. Davis, J. Phys. C $\underline{16}$, L5211 (1983).

${ }^{13}$ B. Derrida and J. Vannimenus, J. Phys. A $\underline{15}$, L557 (1982).

${ }^{14}$ S. Alexander and R. Orbach, J. Phys. (Paris) Lett. 43, L625 (1982).

${ }^{15}$ M. Daoud, J. Phys. (Paris) Lett. 44, L925 (1983).

${ }^{16}$ V. Privman and I. Vagner, Z. Phys. B $\underline{50}, 353$ (1983). 